\title{
Clinical analysis: 13 cases of pregnancy complicated with Takayasu arteritis
}

\author{
Yingjia Zhang, Yanna Li ${ }^{1,2}$, Jun Zhang ${ }^{1,2}$ \\ ${ }^{1}$ Department of Obstetrics and Gynecology, Beijing Anzhen Hospital, Capital Medical University, China \\ ${ }^{2}$ Beijing Institute of Heart, Lung and Blood Vessel Diseases, Beijing, China
}

\begin{abstract}
Objectives: To investigate the clinical features, disposition, and effect of pregnancy complicated with Takayasu arteritis (P-TA) on maternal and fetal outcomes.

Material and methods: The clinical data (diagnosis and treatment, peri-pregnancy monitoring, and pregnancy outcomes) of patients with P-TA treated in our hospital between September 2007 and April 2016 were analyzed retrospectively.

Results: Among the 13 P-TA cases, seven were diagnosed before pregnancy, and six were diagnosed during pregnancy; six cases were diagnosed as the generalized type, and seven cases were diagnosed as the cephalic-brachial type; six cases were in the stable stage, and seven cases were in the active stage. All the cases in the active stage underwent glucocorticoid therapy. Four cases developed complications, including cardiac dysfunction combined with preeclampsia in two cases, preeclampsia in one case, and stroke in one case. Eleven patients successfully delivered (nine cases of full-term delivery and two cases of premature delivery); one patient had late miscarriage; one patient had missed abortion. All the parturients survived and delivered 11 neonates (nine full-term neonates and two premature neonates) and one low-birth-weight neonate; no neonatal asphyxia or death occurred.
\end{abstract}

Conclusions: Patients with P-TA can have better maternal and child outcomes through timely diagnosis and treatment, dynamic monitoring, or timely pregnancy termination.

Key words: pregnancy, Takayasu arteritis, treatment, prognosis

Ginekologia Polska 2017; 88, 12: 654-661

\section{INTRODUCTION}

Takayasu arteritis (TA) refers to a chronic progressive non-specific inflammatory disease in the aorta and its main branches, and the lesions are more commonly seen in the aortic arch and its branches, followed by the descending aorta, abdominal aorta, and renal artery; the involved vessels can present full-thickness arteritis, which leads to vascular wall thickening, stenosis, and fibrosis [1-3]. Its etiology is not clear yet and may be related to infection and other factors caused by an immune damage [4]. This disease occurs more commonly in young women [5], and the male to female sex ratio is 1:4; approximately $90 \%$ of the patients are younger than 30 years, and those older than 40 years have a rare onset of this disease. The prevalence of TA in foreign countries is approximately 2.6 in every 1 million individuals $[4,5]$. Because this disease is mainly seen in women at the childbearing age; the patients have an extremely strong pregnancy request $[6,7]$; and even some patients are diagnosed with this disease for the first time, our attention was focused on whether patients with TA can conceive and on how to manage the mother and child to achieve good outcomes. Previously, the understanding of pregnancy related to TA mainly comes from domestic and foreign case reports or small-number case reports, in which most cases are in the stable stage; the number of cases in the active stage is low; and cases delivering in the active stage are rare. TA may occur with peri-pregnancy hypertension, heart failure, cerebrovascular accidents, fetal growth restriction, or even fetal death [5-10]; thus, we focused on whether patients with TA can conceive and on how to manage the mother and child to achieve good outcomes [8]. There has been no systematic and effective report regarding peri-pregnancy monitoring or

Corresponding author:

Yanna Li

Department Obstetrics and Gynecology, Beijing Anzhen Hospital, Capital Medical University

No. 2 Anzhen Road, Chaoyang District

Beijing 100029, China

tel.: (86) 1084005467 fax: (86) 1064456527

e-mail: guangfazhu@126.com 
specific treatment measures targeting patients with TA [11]; therefore, to determine convenient and effective peri-pregnancy treatment measures and monitoring means, we summarized the data of 13 pregnancy complicated with Takayasu arteritis (P-TA) cases treated in our hospital, including seven cases in the active stage of TA, and provided specific and feasible advice for their pregnancy monitoring and relevant management to achieve good maternal and fetal outcomes.

\section{OBJECTIVES}

This study provides a new method of achieving good maternal and fetal outcomes among patients with P-TA and consequently makes pregnancy in such patients possible. The clinical data of these 13 P-TA cases between September 2007 and April 2016 are reported as follows.

\section{MATERIAL AND METHODS General information}

Thirteen patients with P-TA, aged 25-35 years, who were admitted to the Department of Obstetrics and Gynecology, Beijing Anzhen Hospital between September 2007 and April 2016 were enrolled; their mean age was $30.31 \pm 3.22$ years; and their mean age at diagnosis was $27.85 \pm 2.85$ years. Ten patients were primiparas, and three were multiparas; seven patients were diagnosed before pregnancy (Cases 1, 3, 4, 6, 8,9 , and 13). Case 1 underwent descending aorta-abdominal aorta arterial bypass before pregnancy. Case 9 underwent ascending aorta-left carotid artery bypass. Six patients were diagnosed during pregnancy (Cases 2, 5, 7, and 10-12). The patients' general information is shown in Table 1. This study was conducted in accordance with the declaration of Helsinki. This study was conducted with approval from the Ethics Committee of Capital Medical University. Written informed consent was obtained from all participants.

\section{Diagnostic criteria [1]}

All the patients were diagnosed using the classification criteria issued by the American Rheumatology Association in 1990: 1) age at onset: $\leq 40$ years: symptoms or signs appearing before the age of 40 years; 2 ) intermittent limb dyskinesia: one or more limbs, especially the upper limb(s), experience gradually increased fatigue and muscle discomfort when doing activities; 3) brachial artery pulsation weakening: unilateral or bilateral brachial artery pulsation weakening; 4) blood pressure (BP) difference of $>10 \mathrm{~mm} \mathrm{Hg}$ : systolic pressure difference between the bilateral upper limbs of $>10 \mathrm{~mm} \mathrm{Hg}$; 5 ) subclavian artery or aortic murmur: lateral or bilateral subclavian artery or abdominal aorta murmur; 6) angiographic abnormalities: the first aortic branch or the proximal aorta of the upper/lower limbs exhibits ste- noses or occlusions, often as focal or segmental lesions, not due to atherosclerosis, fibroid dysplasia, or similar causes.

Any case which meets three out of the six above-mentioned criteria can be diagnosed as TA, and the sensitivity and specificity of the diagnostic criteria were $90.5 \%$ and $97.8 \%$, respectively.

\section{Clinical classification [1]}

According to the lesion sites, all the cases were divided into four types:

- Cephalic-brachial type (aortic arch syndrome): the carotid and vertebral arteries experience stenosis and occlusion, which can cause varying degrees of cerebral ischemia; further, the BP in the lateral or bilateral upper limbs was reduced or cannot be detected.

- Thoracic-abdominal aortic type: the lesion is located in the descending aorta and abdominal aorta, causing lower limb weakness, intermittent claudication, hypertension, aortic valve insufficiency, or heart failure.

- Generalized type: the patient has a combination of the two abovementioned types, involving multiple lesions; most patients have severe lesions.

- Pulmonary artery type: the lesions are combined and located in the pulmonary artery, and pulmonary hypertension may occur. Approximately $25 \%$ of patients with pulmonary artery type have pulmonary hypertension [12].

\section{Indices for determining the TA stages [13]}

1) Increased erythrocyte sedimentation rate (ESR) and C-reactive protein (CRP) level in the active stage and normal values in the stable stage; 2 ) systemic symptoms, such as fever or myalgia; 3 ) the involved blood vessels develop ischemia and inflammation, such as intermittent limb fatigue, arterial pulse weakening or disappearing, vascular murmur, vascular pain, upper/lower limb BP asymmetry; 4) typical TA angiographic findings. Any patient with two or more initial or aggravated symptoms can be determined as being in the active stage.

\section{RESULTS}

Relationship between glucocorticoid administration and blood CRP level reduction

Among the 13 cases, seven were in the active stage (Cases $2-5,7,11$, and 12); these cases had an increased blood CRP level and underwent glucocorticoid therapy. Cases 2 and 7 presented with heart failure on admission; consequently, their cardiac function was managed, and timely pregnancy termination was initiated. Thus, intravenous hydrocortisone treatment was provided before surgery. On Day 1, their blood CRP levels decreased by $45.2 \%$ and $53.6 \%$, respectively. Cases $3-5,11$, and 12 were orally administered with predni- 


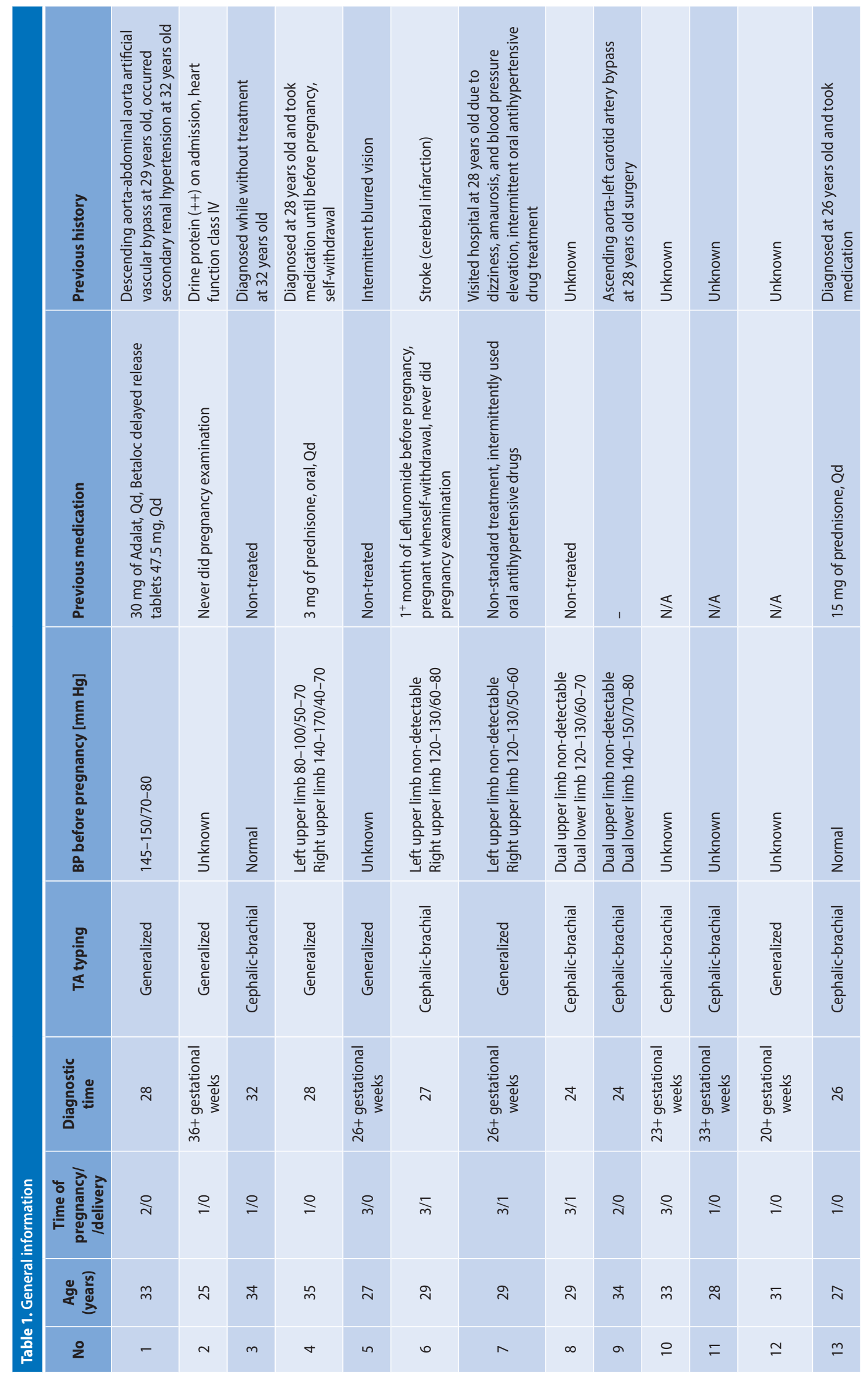




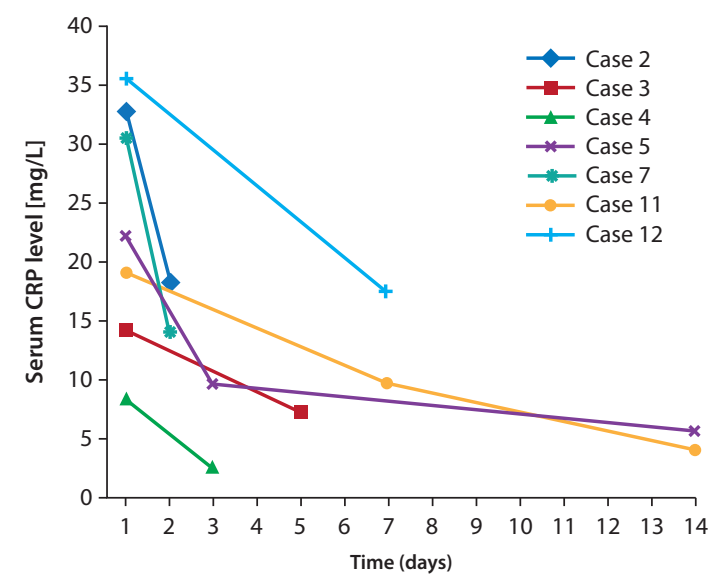

Figure 1. Changes of serum CRP levels after administration of prednisone

sone $(0.75 \mathrm{mg} /$ pre-pregnancy body weight $(\mathrm{kg}) / \mathrm{day})$, and the reduction amplitudes of their blood CRP levels on Days 3-5 were all greater than 20\%. Cases 3, 4, and 12 underwent pregnancy termination owing to their full-term status. Cases 5 and 11 exhibited blood CRP level reduction-to-normal 2 weeks after medication administration, which was maintained thereafter (oral administration of prednisone $2.5 \mathrm{mg} /$ time/day). The pregnancy of Case 5 was maintained until > 35 gestational weeks, and that of Case 11 was maintained until full term and then terminated (Fig. 1).

All the patients in the active stage were intravenously administered with hydrocortisone for 3 days during the perioperative period, which was changed to oral administration of prednisone later (while maintaining the preoperative dosage) and added with cyclophosphamide 1 week after surgery.

\section{Maternal and fetal outcomes}

Among the seven cases in the active stage, two had two complications (Cases 2 and 7), and two had only one complication (Cases 5 and 11). Finally, four patients delivered full-term infants; two patients delivered premature infants (> 35 and > 36 gestational weeks, respectively); and one patient had late abortion. Among all the 13 patients, 11 patients underwent cesarean section in their third pregnancy trimester, including nine full-term deliveries and two premature deliveries ( $>35$ and $>36$ gestational weeks, respectively), and all the infants survived. Among the 11 infants, only one had a low birth weight ( $2270 \mathrm{~g}$ ); all the infants exhibited an Apgar score of 10-10-10, and no neonatal suffocation or death occurred. The maternal and fetal outcomes are shown in Table 2.

\section{DISCUSSION}

TA is a chronic inflammatory vasculitis with an unknown cause, which mainly affects the greater vessels and aorta, as well as its main branches; TA leads to thickening, steno- sis, and fibrosis of the blood vessels [1-3]. Its pathogenesis remains unclear, and existing studies have shown that multiple factors, such as immunity, genetics, infection, or sex hormones, may be involved in TA [4]. Studies have also shown that histocompatibility antigens have a significant correlation with TA [14]; a large number of epidemiological studies have shown that TA dominates in women, with a male to female sex ratio of 1:4. The levels of estradiol and progesterone in patients in either the active or stable stage are higher than those in healthy women, indicating that the elevated levels of sex hormones may be an important factor that can affect the pathogenesis of TA. In addition, TA may be related to chronic antigen stimuli in Mycobacterium tuberculosis, which can then induce non-immune-specific responses [4]; this may be the reason why this disease is more common in Southeast Asia, Central and South America, or Africa [5]. The incidence of TA is low, and more patients are aged $<30$ years, i.e., women at the childbearing age; such patients have an extremely strong pregnancy request $[6,7]$, and even some patients are diagnosed with this disease for the first time. As such, we focused on whether patients with TA can conceive and on how to manage the mother and child to achieve good outcomes [8]. According to previous case reports, TA may occur with hypertensive disorders, heart failure, cerebrovascular accidents, fetal growth restriction, or even fetal death during pregnancy [5-10]. Previously, the understanding of pregnancy related to TA mainly comes from domestic and foreign case reports or small-number case reports, in which most cases are in the stable stage; the number of cases in the active stage is low; and cases delivering in the active stage are even rare. There has been no systematic and effective report regarding peri-pregnancy monitoring or specific treatment measures targeting patients with TA [11]; we used convenient and effective peri-pregnancy treatment and monitoring measures and achieved satisfactory maternal and fetal outcomes.

The histological change in TA involves full-layer arterial inflammation or severe vascular stenosis/obstruction in the diseased vessels $[2,3]$, and the aorta is the main vessel involved during its pathophysiologic process; thus, invasion of the pulmonary artery is possible [2], which may even form aneurysms in rare cases $[4,5]$. The pathophysiological changes in TA include ischemia in the distal end of lesions with BP increases in the proximal end. This disease often recurs, and the interior arterial wall may have a granulomatous inflammatory response, together with significant endometrial hyperplasia, fibrosis, and segmental involvement [2]. The indices of determining the disease stages include: 1) CRP level and ESR: important indices of disease stages. The CRP level and ESR increase faster in the active stage and can return to normal when the disease becomes stable; however, the ESR will also increase during pregnancy. Thus, only the 


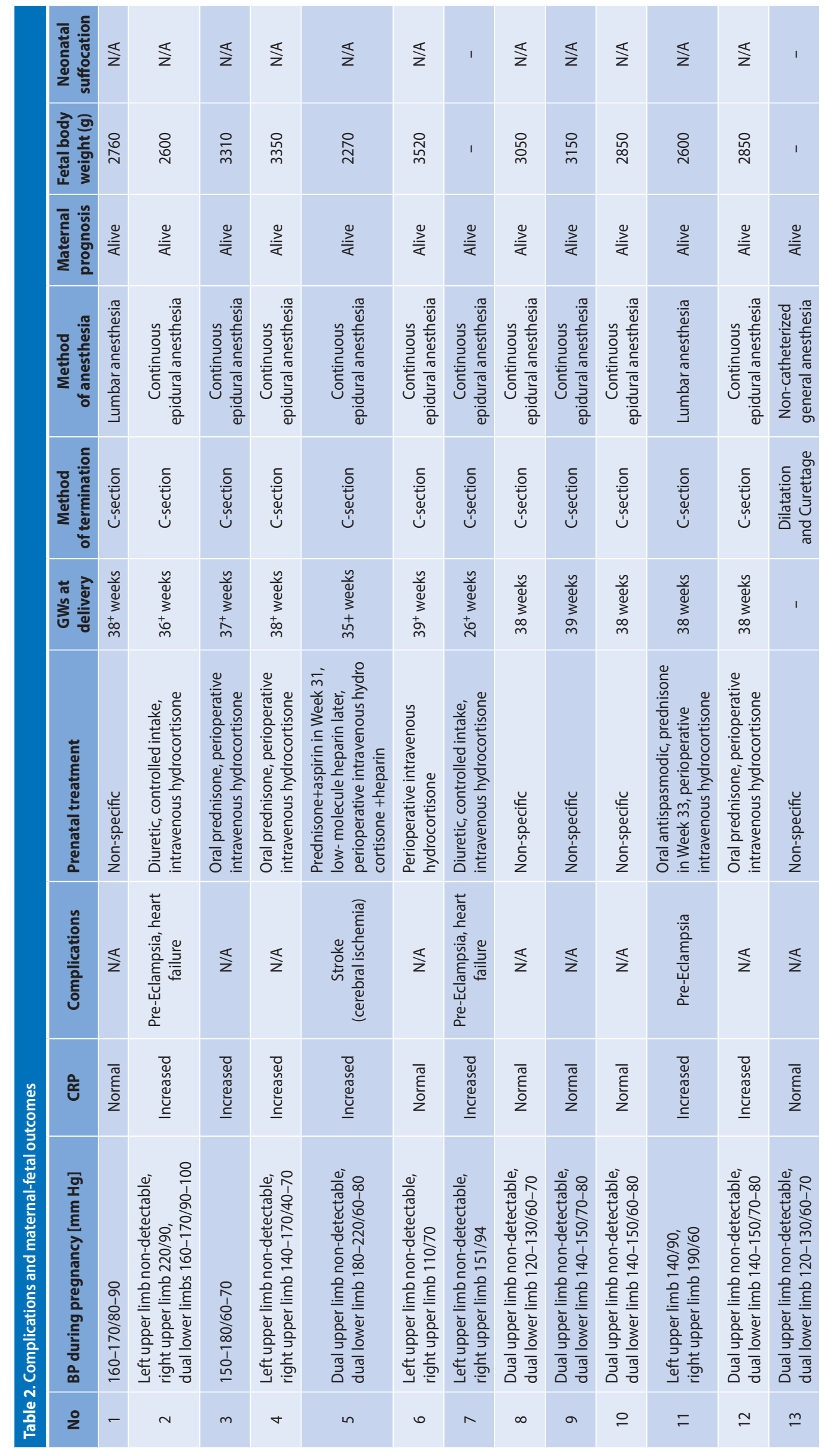


CRP level can be monitored during pregnancy; meanwhile, the effect of other factors, such as trauma or infection, on the CRP level should also be excluded. 2) Some patients may exhibit systemic symptoms, such as fever or myalgia. 3) The involved blood vessels may develop ischemia and inflammation, such as intermittent fatigue, arterial pulse weakening or disappearing, vascular murmur, vascular pain, or upper limb or lower limb BP asymmetry. All the patients treated in our hospital experienced upper limb arterial pulse weakening or disappearing. 4) Patients exhibit typical angiographic TA findings, which directly manifest as changes in the involved vascular lumen, vascular diameter, wall conditions, and length and extent of the involved vessels; however, the thickness changes in the involved vessel wall cannot be shown.

Color Doppler ultrasonography is a useful noninvasive means for assessing vasculitis responses [4] and can explore stenoses or occlusions in the aorta and its main branches (carotid artery, subclavian artery, or renal artery). Any patients with two or more initial or aggravated criteria can be determined as being in the active stage. Taking the effect of contrast agents and radiation on fetuses into account, angiography is not recommended during pregnancy. The ESR also increases during pregnancy; thus, it cannot be used as an effective monitoring indicator, making the CRP level and vascular ultrasound more effective and convenient inspection means during pregnancy. However, as the gestational weeks progress, the use of abdominal vascular ultrasound becomes limited, and related skill requirements and difficulties toward the operators also increase; furthermore, the CRP level is not a TA-specific indicator and can be affected by infection, trauma, or other factors. Hence, the means of TA monitoring during pregnancy are extremely limited.

Pregnancy and TA affect each other. However, most scholars believe that pregnancy is not related to the development of TA [5, 15]; conversely, certain authors proposed that the abovementioned conclusions are based on the data reported [16], which still lack large-sample investigations. Owing to the increase in blood volume, BP can further increase during pregnancy, thus causing preeclampsia, heart failure, cerebrovascular accident, or even death; the placental blood flow will also be affected, causing limited fetal growth or even fetal death [5-7]. At present, most scholars believe that patients with pre-pregnancy TA in the stable stage will have a better prognosis $[6,8,9]$. Conversely, some scholars also believe that there is no significance in the risk of maternal and fetal complications; however, the incidence of such complications is higher in these patients than in normal populations. However, this conclusion still needs further accumulation of relevant cases owing to the small case number reported [7]. Peking Union Medical College Hospital (China) once reported seven cases of TA, six of which had their pre-pregnancy diseases in the stable stage; their pregnancies were all terminated in the late trimester with stable postpartum conditions. One case underwent pregnancy termination in the early trimester, as she was in the active stage, and a cerebrovascular accident occurred, thus exhibiting a poor prognosis. Comarmond et al. [17] retrospectively reviewed 240 pregnancies in 96 patients over 40 years in France and believed that controlling disease conditions before or during pregnancy has an important effect on improving parents' outcomes. Among the 13 patients with P-TA treated in our hospital, six patients were in the stable stage, and seven patients were in the active stage; four patients out of the seven active stage patients developed complications during their pregnancy (57.14\%). The seven patients were administered with glucocorticoids on time, and their CRP level satisfactorily decreased; the CRP level decreased to normal within 5 days in two patients and within 2 weeks in the other two patients (pregnancy was maintained until 35 and 37 gestational weeks, respectively, and was terminated thereafter). Among the seven active stage patients, four delivered in full term; two delivered prematurely (35 and 36 gestational weeks, respectively); and one had late abortion. All the mothers exhibited stable postpartum conditions and were continuously administered with glucocorticoids postoperatively; all of them survived without puerperal infections. Among the six infants, only one had a low birth weight (2270 g); no neonatal asphyxia or death occurred. Among the six stable pregnancies, five were maintained until 37 weeks, and one underwent complete curettage of the uterine cavity because of missed abortion in early pregnancy; all the patients were alive, and no neonatal asphyxia or death occurred. The abovementioned TA data in our hospital also reflect the features of TA (i.e., cases in the active stage had severe disease conditions); however, because the number of cases is limited, further case accumulation is needed for related statistical analyses.

Most women with TA are at the childbearing age; thus, they normally have a strong desire to conceive; considering the abovementioned conditions, we believe that the disease state of such patients before pregnancy should be assessed, and those with stable disease conditions and good organ functions should be considered during pregnancy. However, they also need to be closely monitored [6]. TA-induced hypertension can lead to cerebrovascular diseases and serious complications, such as cerebral hemorrhage or cerebral infarction [18]; hence, treatment against active TA is essential. TA drug treatment can include glucocorticoids, immunosuppressive agents, (cyclophosphamide or methotrexate), biological agents (anti-tumor necrosis factor agents), or anticoagulant drugs [6], among which hormonal drugs are the most important drugs against active TA I [6, 19]. Because immunosuppressive agents and biological 
agents may cause fetal malformations, they are not recommended during pregnancy [6]. When CRP level monitoring in patients with P-TA reveals higher values than normal, the disease condition should be considered in the active stage after ruling out infection, trauma, or other factors, which would require adequate glucocorticoids. The diagnosis and treatment of all the active cases in our hospital were combined with the suggestions from the Department of Rheumatology Immunization: oral administration of prednisone [0.75-1 mg/pre-pregnancy body weight $(\mathrm{kg}) /$ day] and monitoring of blood CRP level; if the CRP level can be decreased by more than $20 \%$ within 2 weeks, the disease control can be considered satisfactory. Consequently, the total dosage of prednisone can be gradually reduced by $5-10 \%$ once; when the dosage of prednisone is reduced to $5-10 \mathrm{mg} /$ day, such a dosage needs to be maintained on a long-term basis. Oral administration of prednisone can be withdrawn perioperatively, and intravenous administration of hydrocortisone can be switched $(200 \mathrm{mg} /$ day for a total of 3 days), followed by oral administration of prednisone (while maintaining the preoperative amount) and gradual reduction every 2 weeks when the disease condition becomes stable; however, the reduction rate should be paid attention to. The active cases in our hospital achieved satisfactory blood CRP level decreases after 3-5 days of oral administration of prednisone; however, their drug reduction rate still adhered to $5-10 \%$ every 2 weeks to avoid a rebound due to a too rapid dosage reduction. Cases 2 and 7 already had heart failure on admission; therefore, their pregnancies need to be terminated as soon as possible. Therefore, they were intravenously administered with hydrocortisone, and their CRP level decreased significantly (45.2\% and $53.6 \%$, respectively). However, owing to the small number of cases, we still need to accumulate such cases further for observation. The risk of glucocorticoids toward mothers is that the body will be in the immunosuppressed state; thus, pregnant women are prone to infection and consequently to gestational diabetes (when combined with insulin resistance increase) $[9,20]$, osteoporosis, pathological fractures, hypokalemia, gastrointestinal bleeding, intraocular pressure increase, or other adverse reactions. Glucocorticoids also need to be administered together with appropriate supplementation of vitamin $\mathrm{D}, \mathrm{Ca}^{2+}$, or $\mathrm{K}^{+}$, as well as electrolyte level, gastrointestinal reaction, or intraocular pressure monitoring. Meanwhile, glucocorticoids also have certain risks toward fetuses and even newborns, which can cause fetal growth restriction, stillbirth, neonatal adrenal insufficiency, or other adverse reactions; hence, fetal growth and intrauterine conditions should be closely monitored during medication administration, and newborns need to be observed to assess for possible adrenal insufficiency. As for anticoagulant drugs, aspirin 75-100 mg/day can be orally administered [19] and changed to subcutaneous injection of low molecular weight heparin 1 week before terminating pregnancy. However, aspirin should be avoided after 32 weeks of pregnancy because it can increase the risk of premature closure of the neonatal arterial catheter and neonatal pulmonary hypertension [20], and the mothers administered with this drug should be monitored via fetal echocardiography. In addition, TA can cause fetal growth restriction or even intrauterine fetal death; fetal growth should then be monitored during pregnancy, together with regular reviewing of fetal ultrasound results and fetal heart rate monitoring. Further, pregnancy should be timely terminated on the basis of the patients' specific circumstances.

Ishikawa and Maetani [21] divided P-TA into four stages and considered kidney diseases, secondary hypertension, aortic regurgitation, and aneurysm as important factors that will affect maternal outcomes: Stage l: free of the above complications; Stage Ila: with one mild complication; Stage Ilb: with one serious complication; Stage III: with more than one complication; aortic regurgitation cases are all divided into Stage III. Vaginal delivery may be considered in patients classified under Stages I and Ila. However, BP increase in the second stage of labor can lead to cerebrovascular accidents; thus, it needs to be shortened. Patients classified under Stages IIb and III should be recommended to undergo cesarean section to terminate pregnancy [10]. When the fetus and placenta are delivered, the placental blood circulation is terminated, and a large volume of blood then reflux and cause great load to the blood vascular system, which can consequently cause cardiovascular and cerebrovascular accidents [18]. BP should be closely monitored during cesarean sections and anesthesia induction to avoid severe BP fluctuations. Currently, epidural anesthesia is more advocated owing to its lesser effect on the hemodynamics [5, 22], and narcotic drugs can be administered in small doses to avoid considerable declines in BP; the brain function can be more easily monitored if the patients can be kept in an awake state, which can also avoid dramatic BP changes stimulated by extubation under general anesthesia $[18,22]$. The 13 pregnancies in our hospital were terminated via surgery; 11 cases were in Stage Ilb or above; 10 cases were induced with continuous epidural anesthesia; two cases with a more stable stage were induced with spinal anesthesia; and one case that underwent complete curettage of the uterine cavity was induced with non-intubated general anesthesia. All the cases were anesthetized and underwent surgery smoothly, and the perioperative periods were uneventful.

Intraoperative intrauterine injection or intravenous application of oxytocin is applicable; it should be used to minimize its effect on the BP [10]. Patients with TA who undergo hormonal therapy may have increased risks of 
postpartum infection [10], and the cases in our hospital were administered with postoperative antibiotics to prevent infection; no puerperal infection occurred in our study. Postpartum patients are in the hypercoagulable state; therefore, they should be administered with anticoagulant drugs as early as possible, especially when the obstetric factors are stable, to avoid thrombosis. Postpartum blood circulation increases; the amount of liquid intake should then be paid attention to and controlled to avoid increasing the cardiac load and the incidence of pulmonary edema or cardiovascular and cerebrovascular accidents $[5,10]$. Postpartum breast-feeding is not suitable, and appropriate rest should be ensured in these patients.

In summary, treatment against P-TA requires close cooperation among the Departments of Obstetrics, Rheumatology, Vascular, Cardiology, and Anesthesiology. Sharma et al. [23] once analyzed the outcomes of 24 pregnancies in 12 patients with P-TA within 20 years, including 17 live neonates (five neonatal growth restrictions and four preterm deliveries), five cases of intrauterine fetal death, and two cases of abortion. In this study, we studied 13 P-TA cases; seven active stage cases were administered with glucocorticoids during pregnancy; achieved significant effects, satisfactory decrease in the CRP level, and stabilized condition; and finally delivered four full-term neonates and two preterm neonates (> 35 and > 36 gestational weeks). There was one case of late abortion. The 13 P-TA cases included 11 cases of mid- and late pregnancy deliveries (nine full-term cases and two premature cases), one case of late abortion, and one case of missed abortion; all the mothers survived. Eleven neonates survived (nine full-term neonates and two premature neonates), including one low-birth-weight neonate. No neonatal asphyxia or death occurred; thus, a satisfactory clinical treatment outcome was obtained. This study provides important clinical data as well as a simple and feasible method for pregnancy monitoring, specific treatment measures, and maternal and fetal outcomes, so that patients with P-TA can have better maternal and fetal outcomes. However, the number of the clinical cases admitted to our hospital is limited, and because the incidence of the disease is low, we still need to accumulate relevant cases in the future for further analyses to obtain more clinical data to provide more effective help for such patients. Therefore, timely diagnosis and close monitoring against TA, timely and adequate glucocorticoid administration against active TA cases, and monitoring of the CRP level as well as the maternal and fetal status to stabilize the disease, together with terminating pregnancy at the appropriate time, can yield good maternal and fetal outcomes.

\section{Conflicts of interest}

The authors declare no conflict of interest.

\section{REFERENCES}

1. Arend WP, Michel BA, Bloch DA, et al. The American College of Rheumatology 1990 criteria for the classification of Takayasu arteritis. Arthritis Rheum. 1990; 33(8): 1129-1134, indexed in Pubmed: 1975175.

2. Gupta S, Chhabra P, Gupta N, et al. Recurrent first-trimester abortion in a young female: Rare presentation of Takayasu arteritis. J Family Med Prim Care. 2016; 5(3): 719-721, doi: 10.4103/2249-4863.197291, indexed in Pubmed: 28217618.

3. Mirault T, Messas E. Takayasu arteritis. Rev Med Interne. 2016; 37(4): 223-229.

4. Johnston SL, Lock RJ, Gompels MM. Takayasu arteritis: a review. J Clin Pathol. 2002; 55(7): 481-486, indexed in Pubmed: 12101189.

5. Nalini S, Santa SA. Takayasu Arteritis with Bilateral Renal Artery Stenosis and Left Subclavian Artery Stenosis in Pregnancy. J Clin Diagn Res. 2015; 9(9): QD07-QD08, doi: 10.7860/JCDR/2015/14371.6485, indexed in Pubmed: 26500964.

6. Machen L, Clowse MEB. Vasculitis and Pregnancy. Rheum Dis Clin North Am. 2017; 43(2): 239-247, doi: 10.1016/j.rdc.2016.12.005, indexed in Pubmed: 28390566.

7. Gudbrandsson B, Wallenius M, Garen T, et al. Takayasu Arteritis and Pregnancy: A Population-Based Study on Outcomes and Mother/Child-Related Concerns. Arthritis Care Res (Hoboken). 2017; 69(9): 1384-1390, doi: 10.1002/acr.23146, indexed in Pubmed: 27813289.

8. Pagnoux $C$, Mahendira D, Laskin CA. Fertility and pregnancy in vasculitis. Best Pract Res Clin Rheumatol. 2013; 27(1): 79-94, doi: 10.1016/j. berh.2013.02.002, indexed in Pubmed: 23507059.

9. Tanaka $\mathrm{H}$, Tanaka K, Kamiya C, et al. Analysis of pregnancies in women with Takayasu arteritis: Complication of Takayasu arteritis involving obstetric or cardiovascular events. Journal of Obstetrics and Gynaecology Research. 2014; 40(9): 2031-2036, doi: 10.1111/jog.12443.

10. de Jesús GR, d'Oliveira ICC, dos Santos FC, et al. Pregnancy may aggravate arterial hypertension in women with Takayasu arteritis. Isr Med Assoc J. 2012; 14(12): 724-728, indexed in Pubmed: 23393708.

11. Miyasaka N, Egawa M, Isobe $M$, et al. Obstetrical management of patients with extra-anatomic vascular bypass grafts due to Takayasu arteritis. J Obstet Gynaecol Res. 2016; 42(12): 1864-1869, doi: 10.1111/jog.13139, indexed in Pubmed: 27718287.

12. Hall S, Buchbinder R. Takayasu's arteritis. Rheum Dis Clin North Am. 1990; 16(2): 411-422, indexed in Pubmed: 1971727.

13. Kerr GS, Hallahan CW, Giordano J, et al. Takayasu arteritis. Ann Intern Med. 1994; 120(11): 919-929, indexed in Pubmed: 7909656.

14. Mwipatayi BP, Jeffery PC, Beningfield SJ, et al. Takayasu arteritis: clinical features and management: report of 272 cases. ANZ J Surg. 2005; 75(3): 110-117, doi: 10.1111/j.1445-2197.2005.03312.x, indexed in Pubmed: 15777385.

15. Hauenstein $E$, Frank $H$, Bauer JS, et al. Takayasu's arteritis in pregnancy: review of literature and discussion. J Perinat Med. 2010; 38(1): 55-62, doi: 10.1515/JPM.2009.120, indexed in Pubmed: 19678743.

16. Seyahi E. Takayasu arteritis: an update. Curr Opin Rheumatol. 2017; 29(1): 51-56, doi: 10.1097/BOR.0000000000000343, indexed in Pubmed: 27748689.

17. Comarmond C, Mirault T, Biard L, et al. French Takayasu Network. Takayasu Arteritis and Pregnancy. Arthritis Rheumatol. 2015; 67(12): 3262-3269, doi: 10.1002/art.39335, indexed in Pubmed: 26315109.

18. Lee EH, Choi E, Ahn W. Application of cerebral oximetry for a parturient with Takayasu's arteritis undergoing cesarean section -a case report-. Korean J Anesthesiol. 2013;65(2): 158-162, doi: 10.4097/kjae.2013.65.2.158, indexed in Pubmed: 24024000.

19. Mirault T, Messas E. Takayasu arteritis. Rev Med Interne. 2016; 37(4): 223-229.

20. Soh MC, Nelson-Piercy C, Soh MC, et al. High-risk pregnancy and the rheumatologist. Rheumatology (Oxford). 2015; 54(4): 572-587, doi: 10.1093/rheumatology/keu394, indexed in Pubmed: 25477056.

21. Ishikawa K, Maetani S. Long-term outcome for 120 Japanese patients with Takayasu's disease. Clinical and statistical analyses of related prognostic factors. Circulation. 1994; 90(4): 1855-1860, doi: 10.1161/01. cir.90.4.1855.

22. Xiao W, Wang T, Fu W, et al. Regional cerebral oxygen saturation guided cerebral protection in a parturient with Takayasu's arteritis undergoing cesarean section: a case report. J Clin Anesth. 2016; 33: 168-172, doi: 10.1016/j.jclinane.2016.02.036, indexed in Pubmed: 27555157.

23. Sharma BK, Jain S, Vasishta K. Outcome of pregnancy in Takayasu arteritis. Int J Cardiol. 2000; 75 Suppl 1: 159-162, indexed in Pubmed: 10980356. 